Christiane Geiser

Verkörperte Träume
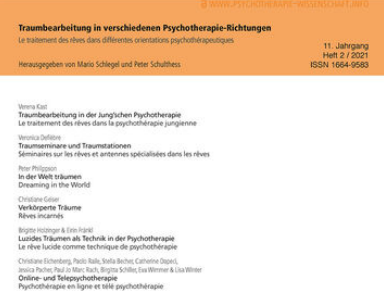

\section{Psychotherapie-Wissenschaft}

11. Jahrgang, Nr. 2, 2021, Seite 47-54

DOI: 10.30820/1664-9583-2021-2-47

Psychosozial-Verlag 


\section{Impressum}

Psychotherapie-Wissenschaft

ISSN 1664-9583 (Print-Version)

ISSN 1664-9591 (digitale Version)

11. Jahrgang Heft $2 / 2021$

https://doi.org/10.30820/1664-9583-2021-2

info@psychotherapie-wissenschaft.info

www.psychotherapie-wissenschaft.info

\section{Herausgeber}

Schweizer Charta für Psychotherapie in der Assoziation

Schweizer Psychotherapeutinnen und Psychotherapeuten

Geschäftsstelle ASP

Riedtlistr. 8

CH-8006 Zürich

Tel. +41432689300

www.psychotherapie.ch

\section{Redaktion}

Mara Foppoli, Lugano

Lea-Sophie Richter, Zürich

Mario Schlegel, Zürich

Peter Schulthess, Zürich

Hinweise für AutorInnen befinden sich auf der Homepage der Zeitschrift: www.psychotherapie-wissenschaft.info

\section{Verlag}

Psychosozial-Verlag

Walltorstr. 10

D-35390 Gießen

+49642196997826

info@psychosozial-verlag.de

www.psychosozial-verlag.de

\author{
Abo-Verwaltung \\ Psychosozial-Verlag \\ bestellung@psychosozial-verlag.de
}

\section{Bezugsgebühren}

Jahresabonnement 44,90€ (zzgl. Versand)

Einzelheft $24,90 €$ (zzgl. Versand)

Studierende erhalten gegen Nachweis $25 \%$ Rabatt.

Das Abonnement verlängert sich um jeweils ein Jahr, sofern nicht eine Abbestellung bis acht Wochen vor Ende des Bezugszeitraums erfolgt.

ASP-Mitglieder wenden sich wegen des Abonnements bitte direkt an die ASP.

\section{Anzeigen}

Anfragen zu Anzeigen bitte an den Verlag:

anzeigen@psychosozial-verlag.de

Es gelten die Preise der auf www.psychosozial-verlag.de einsehbaren Mediadaten.

ASP-Mitglieder wenden sich bitte direkt an

die ASP-Geschäftsstelle: asp@psychotherapie.ch

\section{Digitale Version}

Die Zeitschrift Psychotherapie-Wissenschaft ist auch online einsehbar: www.psychotherapie-wissenschaft.info

\section{(C) $(1) \Theta$}

Die Beiträge dieser Zeitschrift sind unter der Creative Commons Attribution-NonCommercial-NoDerivs 3.0 DE Lizenz lizensiert. Diese Lizenz erlaubt die private Nutzung und unveränderte Weitergabe, verbietet jedoch die Bearbeitung und kommerzielle Nutzung. Weitere Informationen finden Sie unter: creativecommons.org/licenses/by-nc-nd/3.0/de 


\title{
Verkörperte Träume
}

\author{
Christiane Geiser \\ Psychotherapie-Wissenschaft 11 (2) 2021 47-54 \\ www.psychotherapie-wissenschaft.info \\ CC BY-NC-ND \\ https://doi.org/10.30820/1664-9583-2021-2-47
}

\begin{abstract}
Zusammenfassung: Vorgestellt wird der Umgang mit Träumen in einer personzentriert-experienziellen Körperpsychotherapie, wie sie im Ausbildungsinstitut GFK in Zürich vermittelt wird. Nach einführenden therapietheoretischen Überlegungen wird anhand einer ausführlichen, kommentierten Falldarstellung gezeigt, wie der Therapieverlauf unter Einbeziehung des Traumerlebens konsequent immer wieder zum Bezugsrahmen der Klientin zurückkehrt und ihre innere Bewegung in Bezug auf ihr eigenes Erleben unterstützt. Dabei werden unterschiedliche Modalitäten angefragt und ausgefaltet, die der Klientin möglich sind und die den Prozess fortführen. Die Therapeutin ist in der Beziehung als Person mit ihren eigenen Erlebensresonanzen präsent.
\end{abstract}

Schlüsselwörter: Traum, Rogers, Gendlin, Erleben, Prozess, Bewegung, physischer Körper, situationaler Körper, Focusing, Felt Sense

\section{Träume in der personzentriert-experienziellen Körperpsychotherapie}

Träume hatten zu allen Zeiten und in allen Kulturen einen hohen Stellenwert. Wie in diesem Heft ersichtlich ist, wird je nach Konzeptualisierung des Traumgeschehens in den verschiedenen psychotherapeutischen Verfahren sehr unterschiedlich damit umgegangen.

Kennzeichnend für personzentrierte Psychotherapieverfahren, die mit Carl R. Rogers (1940) ihren Anfang nahmen, ist gerade die Abwesenheit einer Apriori-Bedeutsamkeit von Themen oder Inhalten, die es in der Therapie zu beachten gäbe - das gilt auch für die Träume. TherapeutInnen werden ausserdem nicht als «ExpertInnen» angesehen für eine Deutung oder Interpretation der Inhalte. Im Vordergrund steht als Wirkfaktor die Beziehung zwischen TherapeutIn und KlientIn und die Beziehung der KlientInnen zu sich selbst und zu anderen. Eine «Gleichbehandlung» der Themen heisst, dass unterschiedslos allem, was zur Welt der KlientInnen gehört, mit der gleichen Aufmerksamkeit, dem gleichen Interesse und dem gleichen Verzicht auf Bewertung und Lösungsorientiertheit begegnet wird. Nur die KlientInnen können diejenigen sein, die über ihr Leben (und ihr Innenleben!) Bescheid wissen und sich darin auskennen. Es gibt, schreibt Rogers (\& Rosenberg, 1980, S. 179), «ebenso viele «wirkliche Welten» wie es Menschen gibt». In der therapeutischen Beziehung bemühen sich also die TherapeutInnen, den KlientInnen in ihre individuelle Welt zu folgen, sie in ihrem Kontext, ihrem Bezugsrahmen zu verstehen. Sie tun dies aufmerksam und interessiert, um Empathie und Akzeptanz bemüht, im Kontakt mit ihren eigenen Resonanzen, um diese bei Bedarf aufrichtig der Beziehung zur Verfügung stellen zu können. Das war und ist für uns immer noch - eine radikale Position.

Eugene T. Gendlin war ursprünglich Philosoph und ab 1953 Kollege und Mitarbeiter von Rogers in dessen
Forschungsgruppe an der Universität Chicago. Innerhalb der personzentrierten und experienziellen Richtungen ist er vor allem durch seine «Focusing-orientierte Psychotherapie» bekannt geworden. Er legte zusätzlich zu der spezifischen Beziehungsgestaltung im personzentrierten Ansatz vor allem Wert darauf, KlientInnen darin zu unterstützen, sich auf eine spezifische Art und Weise auf ihr Erleben («experiencing») zu beziehen. Das ermöglichte ihnen immer wieder ein neues «Vorantragen» ${ }^{1}$ der gesamten persönlichen Situation und ihrer Bedeutung für ihr Leben. Brachten KlientInnen diese Fähigkeit mit oder konnten sie sie während der Therapie erlernen, erwies sich das in der Forschungsgruppe als hoch wirksam für den Therapieerfolg. Gendlin ging es vor allem um das Wie, also um die Art und Weise des Erlebens («the manner of experiencing»), nicht um das Was (Inhalte, Themen). Seine prozesstheoretischen Überlegungen haben den personzentrierten Ansatz massgeblich beeinflusst.

Die BegründerInnen des GFK ${ }^{2}$ haben den personzentriert-experienziellen Ansatz auch für ihre körperpsychotherapeutische Zugangsweise als Grundlage gewählt. In unserem Ansatz deuten oder interpretieren wir Träume unserer KlientInnen nicht, da nur diese mit Sicherheit sagen können, was in ihnen vorgeht. Allerdings sind auch Träumende selbst nicht dagegen gefeit, dass sie ihren Träumen gegenüber voreingenommen sind. Das zeigt sich an der Art und Weise, wie sie sich an ihre Träume erinnern und sie dann einordnen, interpretieren, an ihr Leben anlegen.

1 Das Focusing-Fachwort «carrying forward» wird hier mit «vorantragen» übersetzt. Es bezeichnet eine spezifische Bewegung im Dialog zwischen dem Erleben und dessen Symbolisierung, die der gesamten Situation eine neue Bedeutung gibt und sie somit «voranträgt".

2 Das Ausbildungsinstitut GFK - Gespräch, Focusing, Körper wurde 1989 von Ernst Juchli und Christiane Geiser gegründet und 1993 in die Schweizer Charta für Psychotherapie aufgenommen. 
Gendlin (1986) und Purton (2006a, b) weisen darauf hin, dass der wichtigste Zeitabschnitt, um uns mit Träumen zu befassen, der zwischen dem Schlaf und dem Wachzustand ist. Purton stellt sogar die Hypothese zur Diskussion, dass wir in dieser Übergangsphase Träume erst kreieren. Je schneller wir zu unserem alten, gewohnten und stereotypen Denken und Fühlen zurückkehren, umso eher sind wir geneigt, Träume mit unseren üblichen Interpretationsmustern zu vereinnahmen.

Wenn wir uns den Träumen unserer KlientInnen in der Therapie wieder nähern, ist es immens wichtig, dass wir das auf eine Art und Weise tun, die diesbezüglich über eine weitere Wiederholung hinausgeht. Deshalb schlagen experienzielle Verfahren in der Nachfolge Gendlins vor, sich beim therapeutischen Umgang mit Träumen auf ein spezifisches körperliches Empfinden, den sogenannten «Felt Sense» zu beziehen, der zur Überprüfung dienen kann, ob wirklich neue kleine Annäherungen entstehen.

\section{Felt Sense}

Gendlin hat schon früh über das Phänomen nachgedacht, dass man manchmal am Morgen aufwacht und "weiss», dass man geträumt hat, aber der Traum ist nicht mehr «da». Was aber zurückbleibt, ist ein «komisches Gefühl im Körper» (Gendlin, 1999, S. 92), und wenn man bei ihm verweilt, kommt der Traum oft zurück. Wir alle kennen ja bspw. auch das Gefühl, einen Raum zu betreten und die Atmosphäre unspezifisch und doch deutlich wahrzunehmen, ohne dass man sie schon benennen könnte. Gendlin nannte dieses spezifische Gefühl «felt meaning» und später "felt sense». Und weil sich die ganze Komplexität dieser gefühlten Bedeutung in verschiedene Modalitäten hinein entwickeln kann, wenn man sich ihr auf eine bestimmte Art und Weise zuwendet, ist das Pendeln zwischen dem Felt Sense und den daraus entstehenden Symbolisierungen für das Überprüfen und Weiterentwickeln des eigenen Erlebens zentral. Diese innere Bewegung immer wieder neu durchzuführen, ist ein wichtiger und erlernbarer Faktor für ein wachsendes Prozessvertrauen bei KlientInnen und TherapeutInnen.

Um sich diesem vorerst vagen und nur als Ahnung wahrnehmbaren «Etwas» nähern zu können, braucht es einen angemessenen inneren Abstand zum eigenen Erleben, den Verzicht auf schnelle Zuschreibungen und Erklärungen und stattdessen ein geduldiges, ergebnisoffenes Warten. Dann kann sich das unscharfe Gefühl zu «all dem» (zur ganzen Situation, zum Thema, zum Problem) allmählich verdichten. Wir richten unsere Aufmerksamkeit auf dieses "Etwas», befragen es auf eine bestimmte Art und Weise, und dann kann es beginnen, sich zu entfalten. In Gendlins Sprache: Etwas Implizites beginnt explizit zu werden. Prozesse können durch diese zyklische Bewegung des «carrying forward» vorangetragen werden.

Wichtig: Gendlins Theorie ist keine Abbild-Theorie, sie hat nichts mit Repräsentationen zu tun oder mit Gleichsetzung. Das, was im Laufe eines Focusing-Prozesses explizit wird, war nicht vorher implizit irgendwo verborgen oder versteckt oder dem Bewusstsein nicht zugänglich. Es war lediglich als Potenzialität vorhanden und wird erst durch eine bestimmte Art der Beziehungsaufnahme und durch diesen Prozess des Zugewandtseins ins "Dasein» geholt.

Auf den Umgang mit Träumen angewendet, schlug Gendlin in seinem (lesenswerten!) Buch Let your Body interpret your Dreams (1986) ${ }^{3}$ und in späteren Artikeln (Gendlin, 1992; 1996; 1999; 2012) vor,

$>$ als Einstieg in die Traumarbeit alle Fragen, die wir aus anderen Verfahren kennen, zu verwenden (z.B. «Was am Traum ist denn das Wichtigste?», «Erzähl den Traum in drei Schritten.» oder «Sei du dieser Teil des Traums.»), aber sie sollen lediglich als Einladung an den Felt Sense dienen und sofort wieder verlassen werden, wenn sich im Erleben nichts Neues bewegt;

> eine «Voreingenommenheitskontrolle» («bias control») einzubauen, die helfen soll, eigene eingeschränkte Deutungsmuster zu erkennen und sich zum Beispiel zu fragen: «Und was wäre das Gegenteil davon?», um dann beide Bedeutungen mit dem Felt Sense auf neue kleine Bewegungen zu überprüfen;

$>$ bei schwierigen, angsteinflössenden Trauminhalten Sicherheit und «Hilfe» («life forward energy») suchen zu gehen, indem Träumende Symbole finden, die für sie genügend Kraft haben; indem sie auftauchende Wahlmöglichkeiten ausfalten; indem sie den Felt Sense zum Traum weiterführen: «Wie sollte die Situation stattdessen weitergehen? Was könnte/sollte passieren?» ${ }^{4}$

Träume machen ja nicht nur klarer, wie etwas ist, sondern auch, wie etwas sein könnte. Es geht also immer darum, ob eine der Fragen/Impulse in den KlientInnen etwas hervorruft, das in ihrem Erleben einen Unterschied macht, körperlich gefühlt werden kann und auf einen Wandel hindeutet.

\section{Der Körper in der personzentriert- experienziellen Körperpsychotherapie}

Das Wort «Körper» bedeutet in unserem Ansatz zweierlei: 1. Wir arbeiten mit dem physischen Körper und sind als TherapeutInnen mit unseren eigenen physischen Körpern präsent. Physische Körper atmen, bewegen sich, pulsieren in unterschiedlichen Frequenzen, sind angespannt oder lassen los, tönen, schauen und haben ihre idiosynkratrischen Eigenheiten, die wir als andere Körper mit unserer Resonanz wahrnehmen und beantworten können. Wir arbeiten dialogisch mit Wahrnehmung, mit Berührung, mit Experimenten - und tun das eingebettet in das personzentriert-experienzielle Verfahren, das unserer ganzen Arbeit zugrunde liegt. 2. Vor allem angeregt durch Gendlin verwenden wir aber auch den Begriff «situationaler Körper» («body-

31987 auf Deutsch erschienen: Dein Körper. Dein Traumdeuter.

4 Diese Art des Zugangs hat sich vor allem bei der Arbeit mit traumatisierten Menschen bewährt (Ellis, 2014, 2019, 2020). 
in-situation») und meinen damit, dass Körper und Umwelt in unauflösbarer Interaktion, in stetigem $\mathrm{Zu}$ sammenspiel sind und das eine ohne das andere gar nicht existieren kann. Nach Gendlin (2016 [1997]) ist der Körper die Wechselwirkung mit seiner Umwelt, er ist unsere gesamte gerade gelebte Interaktion mit der Welt.

Mit unserem situationalen Körper können wir erleben, was für eine Bedeutung eine Situation, in der wir uns befinden, für uns hat. Bedeutung kann jeweils neu entstehen, denn eine Situation ist nie genau so wie eine vorhergehende, auch wenn sie noch so ähnlich ist. Wir speichern in unseren situationalen Körpern aber alle vergangenen Situationen und können versucht sein, eine «alte» Bedeutung in eine «neue» Situation hineinzulesen und dadurch Stereotypie und Starrheit in unseren Lebensprozessen zu erzeugen - Gendlin (1964) spricht dann von angehaltenen Prozessen oder «strukturgebundenen» Interaktionsmustern (siehe auch Gendlin \& Zimring, 1955; Geiser, 2010; Geiser \& Moore, 2014).

Diese beiden Definitionen immer präsent zu halten, hat sich in der Körperpsychotherapie bewährt. «Obwohl der Ort, in dem der Felt Sense auftaucht, der Körper ist, ist das, was auftaucht, die Resonanz auf die Situation, eine unentfaltete Komplexität von Lebensaspekten» (Gendlin, 1999, S. 189). Neues entsteht, kann sich verkörpern und so nachhaltig im Erleben einer Person präsent bleiben.

Das folgende Fallbeispiel einer längeren Therapie, in der ein Wiederholungstraum einer Klientin eine grosse Rolle spielt, zeigt eine solche Entwicklung. Die experienzielle Art der Darstellung entspricht dem Ansatz, der im GFK gepflegt wird: nicht nur in der Psychotherapie, sondern auch beim Lesen und Schreiben immer wieder zum «experiencing», dem eigenen Erleben und der eigenen Resonanz zurückzukehren und dies dann in den Lese-und Schreibprozess mitzunehmen. Zwischen den einzelnen Abschnitten der Fallbeschreibung kommentieren theoretische Bemerkungen den Hintergrund unseres Verfahrens.

\section{Kommentiertes Fallbeispiel: «Der Käfigtraum»}

Die Klientin kommt in die Therapie, weil sie sich leer, antriebslos und unlebendig fühlt. Mir fällt auf, dass sie meist reglos auf ihrem Stuhl sitzt, die Beine übereinandergeschlagen. Ihr Körper ist schmal, die Schultern sind hochgezogen und die Arme eng am Körper gehalten, sie macht selten ausgreifende Bewegungen. Die Augen sind oft niedergeschlagen, die Stimme ist leise, die Atmung flach. Meine erste Resonanz: Da lebt jemand, fast ohne $\mathrm{zu}$ atmen, wie unter einer Glasglocke.

In mir könnte ich allerlei denken: Kein Wunder, dass sie sich antriebslos fühlt, wenn sie kaum atmet und sich so stillhält. Und mir fallen vielleicht energetisierende Übungen und Bewegungsvorschläge ein, die ich dann mal machen könnte, um Schwung ins System zu bringen oder die Ladung besser zu verteilen. Ich könnte mich fragen, wo denn die Aggressionen sind. Ich muss mir vielleicht klinische Gedanken über Störungsbilder machen. Es entstehen in mir Bilder und Bewegungen, was ich am liebsten beziehungsmässig mit ihr tun würde: sie im Arm halten, sie schütteln, sie auf die Füsse stellen, ihr den Rücken stärken, ihre Schultern auffalten, ihre Atmung ändern.

All das sind Beispiele, die Rogers unter «ExpertinSein» abbuchen würde: Es schon wissen, wie die Veränderung gehen soll; es sogar besser und früher wissen als die Klientin; Ziele formulieren und abarbeiten; einen Plan für eine Behandlung haben; interpretieren und deuten.

In einem personzentrierten Verfahren, also auch bei uns im GFK, sollen Erfahrung, Ideen und Wissen nicht verschwinden oder gar nicht erst in mir entstehen. Aber sie sollen ein bisschen neben mich gestellt oder im Hinterkopf aufbewahrt werden und warten, ob sie sich im Prozess ergeben, ob wir sie in gemeinsamer Absprache miteinander erforschen wollen und vor allem: ob allgemeine Annahmen (z. B. «blockierte Aggressionen müssen befreit werden») wohl zu dieser Klientin passen, zu ihrer Geschichte, in ihren individuellen Bezugsrahmen.

Was wir stattdessen zu tun versuchen: immer und immer wieder in die Welt der Klientin gehen, sie dort begleiten, von dort aus zu verstehen versuchen, wie es zu dieser Art Ausformung ihres Körpers, ihres Lebens, ihrer Themen gekommen ist; wie es sich jetzt in ihrer Situation anfühlt, so zu sein; wie es ist, so mit mir zusammen zu sein; wie sich das, was sie «unlebendig» nennt, wohl anfühlt, was das in ihrer Welt bedeutet. "What do you want this word to mean?», fragte Gendlin manchmal in seiner Methode «Thinking at the Edge». Rogers würde formulieren: «Was bedeutet das in Deiner Welt?» Ich als Therapeutin weiss nur, was "unlebendig» in meiner Welt bedeuten würde, aber ich kann nicht davon ausgehen, dass meine Vorstellung von «unlebendig» der ihren gleicht!

Also sage ich zurück, was ich verstanden habe, um ihr die Gelegenheit zu geben, zu präzisieren, zu korrigieren, ein bisschen mehr zu finden («Ein bisschen mehr als das, was vorher war», wäre dann schon eine erste kleine Focusing-Bewegung.) Ich kann dann mit der Zeit auch so etwas wie «körperliches Zurücksagen» versuchen: «Wenn ich so sitzen würde wie Sie, würde es mir so und so gehen - ist das bei Ihnen ähnlich oder ganz anders?» Auch das darf/muss korrigiert werden durch die Klientin, viele müssen ausdrücklich dazu ermutigt werden, das zu tun, ihre eigene Aufmerksamkeit zu schärfen und vor allem als Richtschnur immer nur ihr eigenes Erleben zu nehmen, nie etwas unüberprüft oder ihren TherapeutInnen zuliebe zu bejahen, oder weil sie meinen, so etwas müsse so sein und sei doch bei allen so.

Das bedeutet in der personzentrierten Praxistheorie der Begriff «Akzeptanz». Ich muss nicht einverstanden sein oder es gut finden, was ich antreffe, aber ich akzeptiere immer zuerst die spezifische Ausformung des Lebendigen, wie sie sich in dieser Person zeigt, ohne sofort etwas ändern zu wollen.

Also versuche ich auch bei dieser Klientin, ihr zuerst in ihrer Welt zu begegnen. Sie wirkt zutiefst erschöpft, 
ihr Wort ist «resigniert». Es ist ihr wichtig, dass ich das verstehe, dass das so sein darf, dass ich bei ihr bleibe, auch in diesem Zustand.

Selten regt sich in ihr ein Wunsch nach Veränderung. Da darf ich nicht drängen! Ich versuche, beides zu halten: die Resignation und diesen kleinen Wunsch. Sie sagt: «Manchmal würde ich gern einfach draufloslaufen. Oder wegfahren.» Es sind unspezifische Bewegungsahnungen, weg von etwas. Sie weiss nicht, wie sie das in ihrem Leben anstellen könnte. Impulse erlöschen sofort, Fragen nach einem allerersten kleinen Anfang im Körper zu einer solchen Weg-Bewegung kann sie nicht beantworten, und sie entstehen auch nicht spontan.

In einer Stunde erinnert sie sich, dass sie schon als Kind in schwierigen und unauflösbaren Situationen ein kleines Mantra vor sich hingemurmelt hat: «weg bloss weg». So alt ist diese Vorstellung also schon. "Aber das konnte ich ja nicht», erläutert sie.

Mit ihr ist das Tempo auffallend langsam. Es gibt über längere Zeiträume nur karge Dialoge, kleine Bögen, viel Schweigen. Manchmal bin ich unruhig - müsste ich mehr tun, anbieten, erkennen? Aber diese Versuchung kenne ich schon ... und ich weiss aus Erfahrung und aus Überzeugung, dass Dabeibleiben und Vertrautwerden mit den Veränderungsvorstellungen und Rhythmen der KlientInnen sich lohnen.

In dieser ersten Zeit beginnen ihre Träume. Sie nennt sie «meine Käfigträume»: Da ist ein Vogel, der sitzt in einem Käfig. Er ist allein in diesem Käfig und sitzt dort auf einer Stange. Dieser Traum wiederholt sich mit Varianten über eine lange Zeit. Auch hier kommt mir natürlich allerlei in den Sinn. Wie das zu ihr passt. Wie es dort wohl ist. Wie es weitergehen könnte. Aber ich will zuerst besser verstehen, was der Traum für sie bedeutet, wie es $i h r$ damit geht.

Der Traum, fällt uns beiden nach einer Weile auf, ist kein Film. Es gibt keine Handlung. Es ist eher ein Foto. Mit Farben? Nein, schwarz-weiss. Der Vogel sitzt immer auf derselben Stange, ganz still, und bewegt sich nicht. Es gibt auch sonst keine Bewegung in dem Bild. Sie hat auch keine Ahnung, wer den Vogel versorgt, Wasser und Körner und Vogelsand sind auf dem Bild zu erkennen, aber es gibt keine Veränderung in der Zeit, sodass mal mehr oder mal weniger Futter oder Wasser in den Behältern ist oder dass jemand kommt und es bringt, evtl. sogar spricht. Sie schüttelt den Kopf, da ist niemand. Das Bild/der Traum ist wie eingefroren, wie ausgeschnitten aus einer Abfolge, und wiederholt sich einige Zeit lang genauso immer wieder. Das Gefühl «So ist es halt» (in ihrem Leben, nun auch in ihrem Traumbild) bleibt unverändert.

Gendlin (1964) hat das «frozen wholes» genannt, eingefrorene Ganzheiten, wie Eisstücke, die in einem Strom mitschwimmen, sich aber nicht verändern und immer gleichbleiben, unabhängig davon, wie die Umgebung ist.

In einer der folgenden Stunden entscheide ich mich, vorsichtig eine der Anregungen, die aus anderen therapeutischen Ansätzen bekannt sind, auszuprobieren, wie es Gendlin in seinem Traumbuch vorschlägt, nämlich einzelne Aspekte des Traums herauszugreifen und ihr vorzuschlagen, sich vorzustellen, sie zu sein und so zu einem veränderten inneren Erleben zu kommen.

Nachdem sie sich bereit erklärt hat, etwas auszuprobieren, beginne ich mit dem Vogel. Ich frage: «Wie wäre es, wenn Sie sich vorstellen, dieser Vogel zu sein?» «Das ist einfach», sagt sie sofort, «der ist ja ein bisschen wie ich, ich sitze ja auch oft so da, so still.» Was sie sieht? Die Käfigdrähte, sonst nichts. Aha, sie ist jetzt der Vogel, schaut nicht mehr von aussen auf den Käfig und erkennt sofort die Ähnlichkeit mit sich, ihrem körperlichen Dasein. Aber die Antworten sind schnell, der kleine Dialog ist wieder am Ende. Ich erweitere die Frage ein bisschen: «Was würde es denn brauchen in dieser Situation?» Die Frage ist eindeutig falsch oder zu früh oder beides. Sie schaut irritiert. Es ist halt so, sie ist dort gefangen und es gibt keinen Ausweg.

Ich schlage eine zweite Möglichkeit vor: «Wie wäre es, die Stange zu sein, auf der der Vogel sitzt?» «Ah, die Stange. Die habe ich noch gar nicht angeschaut. Die ist aus Holz, rund, an beiden Seiten mit einer Rille im Draht befestigt und kann nicht herausrutschen.» Präzise beobachtet! «Und ist da noch mehr?» «Die Krallen des Vogels passen gut drumherum. Die Stange ist fest und sicher.» - In mir schlägt so etwas wie ein kleiner Gong an ... irgendetwas ist interessant und neu an ihrer Beschreibung. Ich sage: «Und Sie als Stange?» «Ich? Ich bin fest und sicher? Das verstehe ich nicht.» «Oder», frage ich, "was in Ihnen ist so wie diese Stange? Gut befestigt, fest und sicher, dient einem Lebewesen als Aufenthaltsort?» Sie ist ratlos. Aber beim Nachdenken wirkt sie belebter, sie sucht aktiv, unsere Dialoge werden länger. Dort ist etwas Weiterführendes.

Gendlin rät, alle Fragen, die nicht bald zu einer neuen Erlebensqualität und zu einem «carrying forward» führen, fallen zu lassen und etwas anderes zu versuchen. Fragen sollen nur als Anstoss dienen, und wir wissen vorher nie, welche wann im Prozess etwas auslösen. Wichtig ist nur, ob das Erleben zusammenschrumpft oder aufblüht.

Also probiere ich die dritte Möglichkeit auch noch, wir haben noch etwas Zeit: zu versuchen, wie ist es, der Käfig zu sein. Und dieses Mal erkläre ich ein bisschen mehr zu der Art und Weise, wie sie mit dieser Frage umgehen kann: «Könnten Sie versuchen, eine Weile auf die innere Antwort zu warten und die schnellen, bekannten Antworten vorbeiziehen zu lassen? Und alle noch so kleinen Regungen aufspüren, sie ernst nehmen und abwarten, ob sie sich weiter auffalten können?» Neugier, ein Funke. Ja, das will sie versuchen: "Also, ich als Käfig.» Sie versucht, sich hineinzufühlen, schweigt lange, bewegt sich nicht, sitzt wie immer in dieser zusammengefalteten, engen Haltung. Ich frage vorsichtig, ob sie vielleicht körperlich etwas verändern muss, um jetzt dieser Käfig zu sein. Sie schaut auf. «Nein», sagt sie, «das ist ja das Seltsame. Es ist fast kein Unterschied. Es fühlt sich ganz ähnlich an.»

Hier passiert etwas Neues, ich kann es in meiner Resonanz deutlich spüren, etwas Neues in der Art und Weise, wie sie reagiert, was in ihr passiert. Eine kleine Verwunderung, Überraschung, etwas ist «seltsam». Ich 
sage nur zurück: «Ah, das Seltsame ist, dass es gar keinen Unterschied macht, ob Sie der Vogel sind oder der Käfig es fühlt sich im Körper ganz ähnlich an.» «Ja ...», sie wartet und sagt dann, «es ist wie ...», jetzt spricht sie in diesem typischen Stil, den wir in der Arbeit mit Focusing kennen: suchend, tastend, wartend auf mehr Präzision, auf mehr Klarheit. Dann sagt sie ganz ruhig: «Der Käfig hält den Vogel gefangen. Der Vogel ist im Käfig gefangen.» Da ist wieder die neue Qualität. Ich wiederhole den Satz wörtlich und ganz langsam. Und vergesse dann für einen Moment, dass ich nicht zu schnell sein darf, dass sie ja nah genug an «etwas» dran ist, dass es eine Pause gebraucht hätte - einfach abwarten -, und schicke hinterher: «Wenn Sie Ihren Worten hinterherhören, was gibt es für ein Echo in Ihnen?» Sehr schnell fragt sie: «Bedeutet das, ich halte mich gefangen? Ich mich selbst? Ich bin also selbst schuld daran?» Ach - schade. Das tastende Suchen, die Prozessverlangsamung ist unterbrochen, und schnell taucht wieder eine quasi logische Schlussfolgerung auf, ein Deutungsversuch, eine Interpretation in ihrem üblichen Rahmen, ein Selbstvorwurf. Ich ärgere mich über mich selbst.

Da die Stunde zu Ende ist, bitte ich sie, ob sie diese Sätze und Fragen in sich halten kann, ohne gerade eine Antwort zu haben oder eine Vorannahme, nur in der Nähe bleiben, $a b$ und zu dort hinspüren. Sie will es versuchen und geht nachdenklich weg.

In die nächste Stunde kommt sie ungewöhnlich erregt und erzählt, dass sich das Traumbild völlig verändert habe: Ich sitze als Vogel auf meiner Stange im Käfig - aber das Käfigtörchen steht offen! Jetzt gilt für mich: Vorsicht, nicht irgendeinen Vorwärtsschritt antizipieren, mich sofort freuen oder sie gar ermutigen, rauszufliegen! Ich weiss noch nicht, wie das für sie ist, diese Veränderung, sehe nur, dass sie unruhig ist, fast ein bisschen geschockt aussieht. Also richte ich eine Frage an ihr Erleben im Moment: «Wenn Sie der Vogel sind und Sie sehen, dass die Tür offensteht: Wie ist denn das jetzt gerade für Sie?» Sie beginnt zu weinen, zum ersten Mal, seit ich sie kenne: «Sie meinen jetzt sicher auch, ich könne ja einfach rausfliegen, nicht wahr? Das wäre ja auch normal - aber ich kann nicht! Wieso kann ich das denn nicht?» Sie wirkt ganz verzweifelt, aber «verzweifelt» ist mein Wort, also frage ich nach dem Gefühl jetzt gerade. «Ich fühle mich so hilflos! Ich müsste das doch können, es ist ja nichts mehr im Weg! Wieso kann ich das denn nicht, einfach rausfliegen?»

Das ist eine wichtige Stelle. Zum ersten Mal deutliche Emotionen, zum ersten Mal bezieht sie mich als Person, die etwas über sie denken könnte, mit ein: eine Annahme, dass ich sie bewerte, so wie sie sich selbst auch bewertet in ihren Fragen. Ich erkläre ihr den Unterschied zwischen einer wirklichen Frage und einer anderen Art Frage, die zwar wie eine klingt, aber keine ist, sondern eher ein Vorwurf, eine Bezichtigung, ein Anspruch. Sie versteht sofort: «Ah ja - also, wenn ich mich einfach frage <Wieso kann ich es nicht?, gäbe es vielleicht eine Antwort? Oder mehrere?» Genau, darum geht es. Sie wartet und stellt sich offenbar innerlich diese Frage noch einmal, ergebnisoffen.
Dann sagt sie: «Die Antwort ist: Ich weiss es nicht. Ist das eine Antwort?» Oh ja, das ist eine Antwort. Sie kam dieses Mal aus ihrem Erleben.

Ihre Stimmung hat sich verändert. Sie fragt dann doch noch einmal mich direkt: «Finden Sie denn, ich müsse das können, dort rausfliegen, weil ja die Tür offen ist? Vielleicht geht sie wieder zu und ich verpasse den Moment?» Ich antworte aufrichtig, dass ich nicht weiss, was für sie richtig ist, dass wir alle nur selbst herausfinden können, welche Variante einer Situation uns im Moment möglich erscheint und welche nicht, und dass sie sich dafür Zeit lassen darf und ich definitiv nicht denke, dass sie etwas «können müsse». Sie bedankt sich, dass ich ihr antworte.

Wir zwei Nicht-Wissenden suchen gemeinsam weiter, bleiben in den nächsten Stunden eine Weile bei den Themen Gefangenschaft und Sicherheit, und ich ermutige sie, diese Wörter an ihrem Erleben festzumachen, nicht als Worthülsen hin- und herzubewegen und sich nur gedanklich damit zu befassen. Was ist eine sichere Art zu sitzen, zu liegen, zu stehen? $\mathrm{Zu}$ atmen? Wir probieren, vieles passiert in der Vorstellung. Wann wird es unsicher? Wie fühlt sich Gefangensein an? Ist das Gefühl ähnlich oder ganz anders als das der Sicherheit? Neues kann angespürt werden.

Dann auf einmal kommt sie aufgeregt in die Praxis, ihre Schritte sind schneller und leichter, die Augen weit offen. Der Traum hat sich völlig verändert: Käfig und Vogel sind verschwunden, aber von weit weg sieht sie kleine bunte Vögel, die herumfliegen. Oh, ein Film? «Ja, sie bewegen sich!» Sie macht die Bewegung ansatzweise mit den Händen, den Fingern. Nein, kein Hintergrund, keine Umgebung, sie hört auch nichts, sieht nur diese kleinen bunten Flattervögel, die manchmal auch ein bisschen näherkommen.

Auf einmal ist sie ganz still. Dann sagt sie: «Das hatte ich ganz vergessen ... Wissen Sie, meine Mutter hatte immer Vögel, zuerst in einem kleinen Käfig, in dem sie allein oder zu zweit waren, Wellensittiche und Kanarienvögel. Sie haben manchmal gesungen oder die Wellensittiche haben miteinander geredet in ihrer Vogelsprache, und wenn sie allein waren, mit einem Spiegel, in dem sie sich sehen konnten. Das waren so Käfige wie der im Traum. Aber das meine ich nicht. Als ich schon nicht mehr zu Hause wohnte, hat meine Mutter in einem verglasten Balkon eine grosse Voliere gebaut und viele kleine exotische Vögel hineingetan, und als ich einmal nach Hause kam, sind die darin herumgeflattert. Sie hatte so viel Freude daran». «Und Sie, Sie auch?» "Ja, ich auch. Die waren viel lebendiger und so bunt.» Sie lächelt. «Es hat mir auch imponiert, dass sie das selbst gebaut hat. Und als ich das nächste Mal nach Hause zu Besuch kam, hatte sie ihnen tatsächlich beigebracht, dass sie im Zimmer überall herumfliegen konnten, aber nachts in ihre Voliere zurückkehrten zum Schlafen. Und nach einigem Üben hat das auch geklappt.»

Hier scheint auf einmal das auf, was Gendlin «Hilfe» nennt, im Traum schon angedeutet und davon angestossen dann in der Erinnerung. Die «life forward energy» ist spürbar. Es ist nicht nur das Motiv der kleinen bunten 
fliegenden Vögel und wie sie zwischen Freiheit und dem sicheren Schlafort wechseln, sondern vor allem die Art und Weise, wie sie davon erzählt. Ein Leuchten ist in ihren Augen, sie atmet leichter, bewegt sich beim Erzählen. Ich bleibe lange mit ihr bei dieser Erinnerung, bin selbst beschwingt, berührt. Wir lächeln zusammen, reden hin und her. Was denn das Schöne gewesen sei, was genau hat sie so gefreut? Dass sie so lebendig gewesen seien, geflattert und gepiepst hätten sie, alle durcheinander. Und so bunt. Und so vielfältig. Lange habe sie ihnen zugeschaut, wie sie hin und hergeflogen sind im Zimmer und neue Plätze erkundet haben. Manchmal sei ein Vogel auch auf ihrem Finger gelandet, den sie geduldig lange Zeit in die Luft gehalten hat. Diese kleinen Krallen, dieses Federgewicht ... sie schluckt und hält ein bisschen an, es berührt sie, all das Leben, das ihr wieder einfällt. Und am Abend seien sie (die Voliere abgedeckt und nur das Törchen einladend offen) tatsächlich wieder zurückgekehrt und seien schlafen gegangen. «Freiwillig aus dem offenen Türchen raus und abends freiwillig wieder rein?», frage ich. Sie lächelt, erkennt das Motiv wieder, raus und rein. Sicherheit und Freiheit.

Was für eine andere Energie in dieser Stunde! Was für Veränderungen an so vielen Orten, auch zwischen uns! Ich bitte sie zum Abschluss, etwas zu diesem gesamten «all das» in dieser Stunde zu finden, eine Art Symbol, ein Bild, einen Satz, eine Bewegung - so etwas wie ein Griff an einem Behälter, an dem sie diese ganze Stunde sozusagen wieder hochheben und sich daran erinnern kann (eine klassische Focusing-Intervention). Sie fühlt sich «bewegt», sagt sie, erleichtert, etwas ist passiert, sie spürt es körperlich, auch wenn sie noch nicht alles versteht. Und dass sie das alles hat vergessen können!? Ihr "Griff» ist das Gefühl der kleinen Krallen und das Gewicht des kleinen Vogels auf ihrem Zeigefinger, sie kann es sofort wieder abrufen. "Wie ein kleiner Zwischenstopp für den Vogel auf einer Entdeckungsreise», sagt sie und lächelt.

Gendlin nennt so eine Erfahrung «felt shift» und Rogers (1961, Kap. 7) «moment of movement». Etwas hat sich eine kleine Runde weiterbewegt, und alles, das Ganze der Situation, ist anders geworden. Erstarrtes fliesst wieder, Spannung löst sich. In Gendlins (2016) Prozesssprache heisst das, dass der Körper den angehaltenen Prozess nicht mehr tragen muss. Das ist eine körpertherapeutisch gesehen andere Art und Weise muskulärer und gewebemässiger Entspannung als durch Auf- und Entladung.

Ein paar Tage später bittet sie mich um eine zusätzliche Stunde, was noch nie vorgekommen ist. Zum Glück kann ich es einrichten. Sie hat einen Zettel in der Hand, den sie mir gibt, darauf steht ein Text. Ihr sei eine Melodie nachgelaufen, die ganze Zeit, und auf einmal sei es ihr eingefallen: ein Beatles-Lied über eine Amsel, und dann sei sie es suchen gegangen und habe den Text abgeschrieben:

Blackbird singing in the dead of night take these broken wings and learn to fly all your life you were only waiting for this moment to arise ...
Ich erkenne es sofort und summe, nicht wahr, das ist es? Wir summen zusammen und singen dann sogar den Text, sie hat eine ganz reine, klare Stimme. Eine neue überraschende Modalität. Dann schaut sie mich an, fragt ganz ernsthaft: "Glauben Sie, dass meine Flügel gebrochen sind? Und ich gar nicht fliegen kann, auch wenn ich es wollen würde?»

Eine richtige Frage. Eine wichtige Frage! Ich will sie nicht nur metaphorisch verstehen, sondern mich auch körperlich dieser Frage zuwenden. "Wollen wir es herausfinden?», frage ich, sie nickt. Und auf einmal ist der Eingang in die Körperarbeit, die in der Vorstellung und sozusagen im Konjunktiv schon ein bisschen begonnen hatte, selbstverständlich und einfach. Wir versuchen vorsichtig, ihre Hände und Arme zu bewegen, die Schultern ein bisschen aufzufalten, ganz langsam, meine Hände geben ihr Halt, wir machen die Bewegungen zusammen. "Nein», seufzt sie erleichtert nach einer Weile, "da ist nichts gebrochen. Aber alles ist so ungeübt, so schwach. Könnten die Flügel wohl stärker werden, kräftiger?» Spielerisch gebe ich ein bisschen Druck auf den Unterarm, sie lacht und versucht, Gegendruck zu geben. «Ach ja, das habe ich auch lange nicht mehr gemacht, wir durften nicht so rangeln mit den anderen Kindern. Und das Auffalten geht auch noch nicht gut. Sie sind ein bisschen eingefaltet, meine Flügel, merke ich, so nach innen, nur nach innen - wie ginge denn das Nach-aussen-Drehen?» Sie versucht zögernd eine andere Richtung, andere Bewegungen. Wir merken, dass das auch im Rest des Körpers etwas verändert, der Brustkorb öffnet sich, hebt und senkt sich, die Atmung wird tiefer. Wir versuchen das alles im Sitzen und dann im Stehen - was das für einen Unterschied macht! Sie wirkt neugierig, interessiert und sehr lebendig.

Diese beiden Stunden sind ein Wendepunkt in der Therapie. Wir arbeiten nun jede Stunde körperlich, nach all den eingefrorenen Stunden geht das erstaunlich mühelos. Gefühle unterschiedlichster Art tauchen auf, Sehnsucht vor allem, mit der Zeit dann auch ein deutliches Wollen, mit geradem Rückgrat. Jetzt kann ich auf mein Repertoire zugreifen und immer wieder Neues anfragen und einladen: «Wo im Rücken wäre Unterstützung gut? Was ändert sich, wenn ich meine Hände hier habe oder hier? Wie viel Druck braucht es?» Gemeinsam erforschen wir neue Bewegungs- und Ausdrucksmöglichkeiten. Ihr physischer Körper verändert sich, sie geht aufrechter, die Augen schauen geradeaus, die Arme schwingen, bewegen sich mit. Mehr Varianten sind möglich.

Manchmal kehren wir zum Vogel zurück: Will der Vogel im Käfig bleiben? Oder nur ab und zu raus und dann wieder rein? Oder nur ab und zu drinnen sein und sonst draussen? Was ist denn überhaupt da draussen? Wie lebt ein Vogel in Freiheit? Sie beginnt sich für Vögel $\mathrm{zu}$ interessieren, wie unterschiedlich sie sind und wie unterschiedlich sie leben.

Vorsichtig entstehen Ahnungen von neuen Wahlmöglichkeiten und Perspektiven in ihrem Leben, die auch ihren situationalen Körper betreffen. Sie rede mehr mit anderen Menschen, sagt sie, sogar am Telefon! Und einmal war sie tanzen, sie, in ihrem Alter! Und dann 
habe sie gelesen, dass es jetzt Interrailtickets auch für Erwachsene gäbe, sogar 1. Klasse, ob sie nicht doch mal probieren sollte zu reisen? Als junge Frau sei sie viel zu schüchtern gewesen. Sie wirkt als ganze Person klarer, mehr im Kontakt mit sich und der Welt. Ich unterstütze sie in all diesen Auffaltbewegungen und auch in ihrem Hin und Her, Sicherheiten nicht aufzugeben. Beides ist wichtig für sie. Der Käfigtraum taucht nicht mehr auf.

Die Therapie geht noch eine Weile weiter und endet dann mit der Zusicherung, dass sie wieder zurückkommen kann, wenn etwas steckenbleibt oder sie diese kleine Vorwärtsbewegung wieder verlieren sollte. Zum Abschied sagt sie: «Fast wäre ich dortgeblieben, in diesem Käfig ... Ich bin so froh, dass ich mich jetzt anders fühle. Mein Leben ist so viel lebendiger geworden.» Und was für sie fast am wichtigsten gewesen sei: dass sie jetzt diese kleine innere Bewegung identifizieren kann, dieses kleine Etwas in ihr, das erst vage da ist, aber doch deutlich genug, dass sie sich darauf beziehen und es anfragen kann. Dann kann sich dieser typische kleine Pendelprozess wieder in Gang setzen, der immer etwas mehr bringt als das, was vorher war, was sie jedes Mal wieder als Erlösung aus der drohenden Erstarrung empfindet. Etwas ist zuverlässig dort am Leben, sie muss es nur immer wieder finden. "Wie ein kleiner Kompass, der mir gehört», sagt sie, «ich kann mich immer an ihn erinnern und ihn suchen gehen - und dann weiss ich wieder ein bisschen weiter.»

\section{Literatur}

Ellis, L.A. (2014). Living the Dream: The Evolution of Focusing-Oriented Dream Work. In G. Madison, Focusing-Oriented Psychotherapy. Innovative Theory and Applications (S. 166-177). London: Jessica Kingley Publ.

Ellis, L.A. (2019). Body dreamwork: Using focusing to find the life force inherent in dreams. International Body Psychotherapy Journal, $18(2), 75-85$

Ellis, L.A. (2020). A Clinican's Guide to Dream Therapy. New York: Routledge.

Geiser, C. (2010). Moments of Movement: Carrying Forward Structure-Bound Processes in Work with Clients Suffering from Chronic Pain. In PCEP, 9(2), 95-106. https://christianegeiser.ch/wp -content/uploads/2017/06/Carrying-Forward-Structure-Bound -Processes.pdf (09.07.2021).

Geiser, C. \& Moore, J. (2014). New Ways of Processing Experience. How to Work with Structure-Bound Processes. In G. Madison (Hrsg.), Theory and Practice of Focusing-Oriented Psychotherapy. Beyond the Talking Cure (S. 130-145). London: Jessica Kingsley Publ. https://christianegeiser.ch/wp-content/uploads/2017/06/ New-Ways-of-Processing-Experience.pdf (09.07.2021).

Gendlin, E. T. (1964). A theory of personality change. In P. Worchel \& B. Donn (Hrsg.), Personality Change (S. 100-148). New York: John Wiley \& Sons. http://previous.focusing.org/gendlin/docs/ gol_2145.html (09.07.2021).

Gendlin, E. T. (1986). Let your body interpret your dreams. Wilmette/ IL: Chiron Publ. ([1987]. Dein Körper. Dein Traumdeuter. Salzburg: Otto Müller.)

Gendlin, E.T. (1992). Three learnings since the dreambook. The Folio, 11(1), 25-30. http://previous.focusing.org/gendlin/docs/ gol_2007.html (09.07.2021).

Gendlin, E.T. (1996). Focusing-oriented psychotherapy: A manual of the experiential method. New York: Guilford Press.
Gendlin, E.T. (1999). Focusing-Traumarbeit. In E.T. Gendlin \& J. Wiltschko, Focusing in der Praxis : Eine schulenübergreifende Methode für Psychotherapie und Alltag. Stuttgart: Klett-Cotta.

Gendlin, E.T. (2012). Body Dreamwork. In P. McNamara \& D. Barrett (Hrsg.), Encyclopedia of Sleep and Dreams. Westport/CT: Praeger Press.

Gendlin, E. T. (2016 [1997]). Ein Prozess Modell. Übers.v. D. Schoeller \& C. Geiser. 2. Aufl. Freiburg: Karl Alber.

Gendlin, E.T. \& Zimring, F. (1955). The qualities or dimensions of experiencing and their change. Counseling Center Discussion Paper, 1(3), 1-27. http://previous.focusing.org/gendlin/docs/gol _2139.html (09.07.2021).

Purton, C. (2006a). Focusing and Dreams. Keynote address at ÖGwG conference Linz, Austria, 27.10.2006.

Purton, C. (2006b). When is the dream-time? Paper presented at the $18^{\text {th }}$ International Focusing Conference, Soesterberg, Netherlands, May 2006. http://www.dwelling.me.uk/WhenDreamTime.htm (09.07.2021).

Rogers, C.R. (1940). Newer Concepts in Psychotherapy. Univ. of Minnesota.

Rogers, C.R. (1961). On Becoming a Person. A Therapist's View of Psychotherapy. Boston: Houghton Mifflin Comp. ([1973]. Entwicklung der Persönlichkeit. Psychotherapie aus der Sicht eines Therapeuten. Stuttgart: Klett Cotta.)

Rogers, C.R. \& Rosenberg, R. (1980). Die Person als Mittelpunkt der Wirklichkeit. Stuttgart: Klett-Cotta.

\section{Embodied dreams}

Abstract: This paper presents an approach to dreams in person-centered, experiential body psychotherapy as taught in the GFK training institute in Zurich. After introductory therapy-theoretical considerations, a detailed, annotated case presentation is used to show how the course of therapy, including dream experience, consistently returns to the female client's frame of reference and supports her inner movement in relation to her own experience. In doing so, different modalities are studied and developed that are possible for the client and that continue the process. The therapist is present in the relationship as a person with her own experiential resonances.

Keywords: dream, Rogers, Gendlin, experience, process, movement, physical body, situational body, focusing, felt sense

\section{Sogni personificati}

Riassunto: Viene presentato un approccio ai sogni tramite una psicoterapia del corpo esperienziale e incentrata sulla persona come viene applicata presso l'Ausbildungsinstitut GFK, Istituto di formazione di Zurigo. Dopo alcune riflessioni introduttive di natura teorica in ambito terapeutico, verrà descritto e commentato esaurientemente un caso che mostra come l'evolversi della terapia che comprende l'attività onirica torni sempre e in modo coerente al quadro di riferimento della paziente e al suo movimento interno con riferimento alla propria esperienza. A questo riguardo, vengono richieste e sviluppate diverse modalità possibili per la paziente facendo avanzare il processo terapico. La terapista è presente nella relazione come persona con una propria dimensione delle esperienze.

Parole chiave: sogno, Rogers, Gendlin, esperienza, processo, movimento, corpo fisico, corpo situazionale, focus, felt sense 


\section{Die Autorin}

Christiane Geiser ist Psychotherapeutin, Supervisorin, Mitbegründerin und ehemalige Ausbildungsleiterin des Ausbildungsinstitut GFK für personzentriert-experienzielle und körperorientierte Psychotherapie in Zürich. Sie arbeitet international als Ausbilderin und Supervisorin und ist zertifizierende Koordinatorin des Internationalen Focusing Instituts in New York.

\section{Kontakt}

E-Mail: christiane.ti@bluewin.ch 